\title{
Comparison of VLBI and GNSS common view for time transfer
}

\author{
Pingli Wang ${ }^{1,2,}$, Guangli Wang ${ }^{1,3}$, Yuping Gao ${ }^{1,2}$, Hongbing $\mathrm{Cai}^{2}$, and $\mathrm{Na} \mathrm{Liu}^{2}$ \\ ${ }^{1}$ University of Chinese Academy of Sciences, Beijing 100049, PR China \\ ${ }^{2}$ National Time Service Center, Chinese Academy of Sciences, Xi'an 710600, PR China \\ ${ }^{3}$ Shanghai Astronomical Observatory Chinese Academy of Sciences, Shanghai 200030, PR China
}

Received: 18 September 2019 / Accepted: 3 November 2019

\begin{abstract}
With the rapid development of optical clock, the stability and system uncertainty of optical clocks has reached a 1.0e-18 level. Optical clocks will likely constitute the next generation of time-frequency standards for redefining the SI second. Because time and frequency transfer services that rely on satellite systems are not always reliable and currently available technologies are insufficient for comparing the next generation of frequency standards, high-precision time and transfer techniques are strongly desired. Very Long Baseline Interferometry $(\mathrm{VLBI})$ is one of the space geodetic techniques that measure the arrival time delays between multiple stations utilizing radio signals from distant celestial radio sources. Not only can VLBI obtain the angle position measurement of the radio source with sub-millisecond accuracy and the station coordinate measurement with millimeter accuracy, but also, it can provide high-precision information regarding interstation atomic clock differences. Therefore, it is theoretically feasible to use the VLBI technology to do the remote time transfer. Because of this characteristic of VLBI technology, VLBI has significant application potential in the field of remote time transfer. To confirm the suitability of VLBI to time-frequency transfer for future practical applications, the results of VLBI and GPS common view time transfer were compared using a Kunming-Urumqi baseline. The performance characteristics of time transfer based on VLBI are then analyzed. Experimental results show that VLBI technology can accurately measure the variation of clock differences between stations as same as the GPS common view time comparison technology. It briefly describes the challenges of future VLBI technology for practical applications of time transfer.
\end{abstract}

Keywords: VLBI / GNSS common view / time and frequency transfer / remote time comparison / experiments

\section{Introduction}

Time is considered to be the most basic physical quantity representing the movement of matter, as well as an important aspect in the development of human civilization. Time is one of the seven basic physical quantities in the international unit system. It is the most accurate and widely used physical quantity among all physical quantities [1]. The definition of the current time unit "second" is based on the atomic clock. High precision time frequency is the basis of modern social and economic developments and scientific research. The unified time standards in the world are International Atomic Time (TAI) and Coordinated Universal Time (UTC). TAI is derived from a combination of data from approximately 500 atomic clocks run by more than 70 time laboratories Two-way Satellite Time and Frequency Transfer (TWSTFT), two completely independent space that maintain the local UTC. TAI is

* Corresponding author: wangpingli@ntsc.ac.cn based on the Global Navigation Satellite System (GNSS) and the technologies for remote time and frequency transfer [1].

GNSS time and frequency transfer based on a navigation satellite (including GNSS one-way time service, GNSS common view (CV), and GNSS precise point positioning (PPP)), and TWSTFT based on communication satellites rely on satellite systems as the primary method for time and frequency transfer. The deterioration of satellite systems-based time and frequency transfer could cause global panic; many electronic systems use satellite navigation systems as the only time transfer method providing a source of time and frequency reference for the system. However, satellite systems have several shortcomings, such as weak signals, vulnerability to interference and attack, limited coverage space, and so on. Because of the important military value of the satellite navigation time service system, there are serious security threats in satellite time service. For example, it is impossible to provide time transfer services in extraordinary circumstances. In 2014, Russia's GLONASS system 
was paralyzed, wherein the timing error exceeded $300 \mathrm{~ns}$. On July 11, 2019, the Galileo satellite navigation system in the European Union broke down, resulting in the disruption of navigation and time service for approximately a week. The GPS and Beidou Satellite Navigation Systems also involve such security risks pertaining to satellite time service. Thus, there are significant security risks in satellite systems.

Moreover, in recent years, great progress has been made in the research of optical clocks at home and abroad; such clocks have promoted the progress of science and technology. Optical clocks have broad application prospects in the precise measurement of basic physical constants, searches for dark matter, gravitational wave detection, and satellite navigation systems. Currently, the stability and system uncertainty of optical clocks based on neutral atoms and single ions has reached a 1.0e-18 level, which is more than two orders of magnitude higher than the existing reference clock-cesium atomic fountain clock [2-5]. Such optical clocks will likely constitute the next generation of time-frequency standards for redefining the SI second. At the 24th Meeting of the General Conference on Weights and Measures (CGPM) in 2011, every National Metrology Institute (NMI) was encouraged to provide resources for the development of optical frequency standards, and to develop new and higher precision timefrequency transfer technologies to assess and compare optical frequency standards worldwide [6]. However, the precision of time and frequency transfer in the commonly used time transfer methods such as TWSTFT and GNSS need to be improved in order to compete with the next generation time-frequency standards. The time transfer precision of the GNSS CV time and frequency transfer has reached approximately the $2 \mathrm{~ns}$ level. Moreover, the time transfer precision of the TWSTFT and GPS carrier phase (CP) time transfer has reached approximately the $0.1 \mathrm{~ns}$ level [7]. To compare the new time-frequency standards with the existing time frequency transfer technologies, average longer periods must be conducted. Advanced highprecision CP TWSTFT technology may provide sufficient precision over long distances, but this depends on the availability and reliability of satellite transponders [8]. The time transfer system based on optical fibers uses closed optical fibers as the transmission media; these fibers have the characteristics of high precision and non-interference, with an associated accuracy of such time synchronization better than $0.1 \mathrm{~ns}$ [9]. Thus, optical fibers may be a particularly promising solution for the comparison and evaluation of modern new time and frequency standards. However, the complex topology of optical fiber networks and the limited space coverage of optical cables still limit the application of this method, although it has recently achieved wire time and frequency transfer between grid nodes across a distance of more than $1000 \mathrm{~km} \mathrm{[10].}$

Because time and frequency transfer services that rely on satellite systems are not always reliable and currently available technologies are insufficient for comparing the next generation of frequency standards, high-precision time and transfer techniques are strongly desired. Therefore, new high-precision time and frequency transfer technologies must be further studied and developed [11-12].
Very long baseline interferometry (VLBI) is a space geodetic technique that obtains the arrival time delays and time delay rate of the radio signal between two antennas by interferometric measurement of the radiation signal from the extragalactic radio sources. Currently, VLBI technology is the astronomical observation technology with the highest angular resolution. Not only can it achieve submillisecond accuracy of radio source angular position measurement and millimeter accuracy of station coordinate measurement, but also, it can estimate the relative information of clock differences between stations with tens of picoseconds of accuracy [13]; this is higher than that of the current time transfer technology using satellite navigation system. This characteristic of VLBI technology gives an important application potential in the field of remote time transfer.

In several recent studies, the ability of VLBI with regard to frequency transfer has been studied. These studies show that VLBI and GPS CP have comparable abilities in frequency transfer in the average period of several hours. However, for shorter average periods, the use of VLBI is limited by its low data sampling rate, which leads to the low time resolution of clock offsets estimation. Therefore, VLBI is not as good as the GPS CP's short-term frequency transmission capability [14]. To confirm the potential of VLBI frequency transfer in future practical applications, National Institute of Information and Communications Technology (NICT) used the KashimaKoganei baseline in 2007 to compare the results of VLBI and GPS CP frequency transfer [15]. Research shows that VLBI is more stable than GPS in frequency transfers between $2000 \mathrm{~s}$ and $6000 \mathrm{~s}$. In 2008, to verify the results of VLBI and GPS CP frequency transfer again, NICT analyzed the comparison results using the International VLBI Service for Geodesy and Astrometry (IVS) data and International GNSS service (IGS) data. The results of VLBI frequency transfer show that the stability is very close to the $1 / \tau$ law, with the stability reaching approximately $2.0 \mathrm{e}-11$ at $1 \mathrm{~s}$ [15].

In 2009, Carsten Rieck of Onsala Space Observatory, Chalmers University of Technology in Sweden and others analyzed the technical characteristics of VLBI timefrequency comparison and conducted experiments using IVS Continuous VLBI Campaign 2008 (CONT08) data. CONT08 is a 15-day continuous VLBI campaign organized by IVS using 11 VLBI stations distributed around the world in August 2008. ONSALA and WETTZELL are also IGS tracking stations, which can achieve time comparison between GPS CP stations. The VLBI time-frequency transfer experiment based on CONT08 involves processing 24-h observation data in batches according to the geodesic mode, during which the clock difference parameter is estimated as a quadratic term with deviation estimates every hour. The results were then concatenated to form 15 consecutive days of time comparison. According to the experimental results, the stability of the VLBI frequency link between ONSALA and WETTZELL station clocks can reach the $1.5 \mathrm{e}-15$ level [14] in one day, which is equivalent to the result of GPS CP. However, VLBI related processors may introduce bias. Daily batch processing of VLBI data results in day boundary offsets which cause 
discontinuity between days, wherein it is easy to overestimate the stability of frequency links. It is also noted that the calibration and monitoring of equipment delay in the VLBI system is the key to time-frequency comparisons using VLBI. In 2012, the results of time-frequency comparison using CONT11 data were basically the same as those of CONT08 [16]. Thus, the ability of VLBI frequency transfer was reconfirmed.

The Chinese VLBI Network (CVN) is composed of 5 radio astronomical telescopes with Shanghai $25-\mathrm{m}$ radio telescopes, Shanghai 65-m radio telescopes, Beijing 50-m radio telescopes, Kunming 40-m radio telescopes, Urumqi 25-m radio telescopes, and Shanghai Data Processing Center. Each observatory station is equipped with a $\mathrm{S} / \mathrm{X}$ band dual-frequency receiver, a VLBI data terminal, a hydrogen atomic clock, and other VLBI observation equipment; stations are upgraded and developed synchronously with developed countries. CVN also actively participates in the international joint measurement activities organized by the European VLBI Network $(\mathrm{EVN})$, IVS, and other organizations. It is an important member of the international VLBI network and has made significant contributions to research in the international VLBI frontier field. Additionally, CVN plays an important role in the Chang' $\mathrm{E}$ project.

Although VLBI has existed for approximately $50 \mathrm{yr}$, there is a little information surrounding remote time transfer using VLBI. To compare the results of VLBI and GNSS CV time transfer, VLBI observation data of Kunming and Urumqi are used to calculate the clock difference between Kunming and Urumqi stations from 2015-2017. The results are compared with the GPS common view time transfer results of Kunming and Urumqi stations.

\section{Materials and methods}

\subsection{VLBI for time transfer}

VLBI technology first appeared in the 1960s, as a kind of space geodesy measurement technology. It has the highest spatial resolution and is widely used in astrophysics, astronomy, geodesy and deep space exploration. The development of VLBI benefits from the availability of highspeed, high density, and large-capacity data storage technology as well as the invention of high-performance hydrogen atomic clocks for the frequency standard $[17,18]$. Before the advent of these technologies, coaxial cables were used to connect multiple radio telescopes and form a connected interferometer. Because the angular resolution of the interferometer is inversely proportional to the baseline length between the radio antennas, the distance between the radio antennas must be increased to improve the resolution of the interferometer. However, in order to achieve this, it is necessary to share the local frequency signal to convert the single received frequency, thus requiring the use of coaxial cables to provide a common local frequency signal to each radio telescope. Additionally, because the received signal cannot be recorded in a reproducible way, it is still necessary to transmit and synthesize the received signal through a coaxial cable. For these reasons, the distance between radio telescopes is

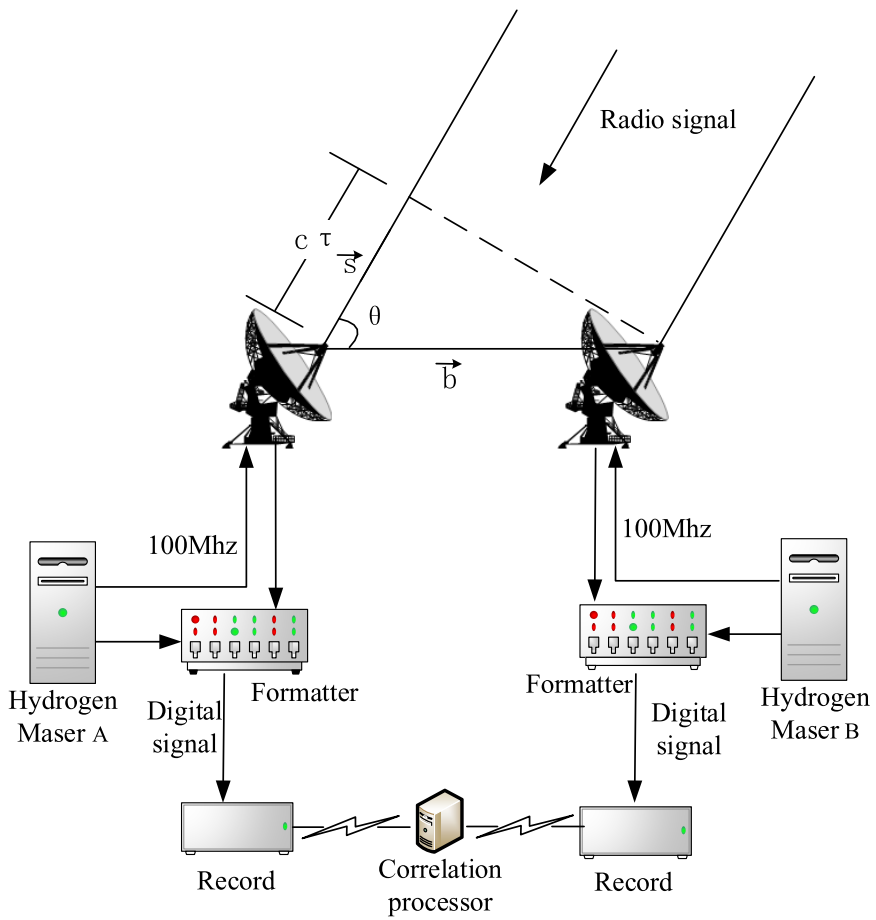

Fig. 1. Measurement principle of VLBI. Here, $\tau$ is the VLBI time delay, $c$ is the speed of light, $\vec{b}$ is the baseline vector, $\vec{s}$ is the Radio source Direction Unit Vector, $\theta$ is the angle between $\vec{b}$ and $\vec{s}$. So $c \tau=|\vec{b}| \cos \theta$.

limited. With the development of hydrogen atomic clocks, local frequency signals using independent frequency standards can be generated for each radio telescope, making it possible to convert frequencies while maintaining consistency. It can also mark the precise time information for the signals, perform Analog/Digital (A/D) conversion at the exact time, and then increase the distance between the radio antennas. VLBI observation is based on the frequency signal of the hydrogen atomic clock output as the reference signal.

The Radio Astronomy and Geodesy VLBI community uses the hydrogen atomic clock at about 50 remote sites around the world, as a sufficient signal-to-noise ratio must be maintained in the process of VLBI observation to maintain the consistency of signal components in the short term. Therefore, a frequency reference with good shortterm frequency stability is needed. It is difficult to maintain frequency stability for a whole day even when using hydrogen atomic clocks with decent short-term stability. Therefore, the clock offset of other observatories and the clock offset of the reference station are estimated every hour, assuming that the clock offset is linear during this period. When the clock offset is estimated hourly, the required frequency stability will be reduced to $2.8 \mathrm{e}-15$ [6]. To achieve approximately 10 degrees signal coherence for approximately $1000 \mathrm{~s}$ at $10 \mathrm{GHz}$, the relative stability of the two clocks at the ends of the baseline need to be maintained at $2.8 \mathrm{e}-14$ within $1000 \mathrm{~s}$. A hydrogen atomic clock provides this level of performance.

The basic measurement principle of VLBI is shown in Figure 1. Two remote observatories receive radio signals 
from the extragalactic radio sources at the same time. The signals received with radio antennas are recorded and marked on a time label using the hydrogen atomic clock. The time delay and time delay rate of the radio signals arriving at the two radio antennas are obtained by interferometric measurement of the radio signals from the extragalactic radio sources. When theoretical delay and propagation path delay are deducted, the difference of arrival time between the two radio antennas can be used to calculate the clock difference between the two stations. This is the basic principle of VLBI time transfer.

The VLBI time delay $\tau$ obtained by the correlation processing is mainly composed of the geometric delay $\tau_{g}$. There are other significant delays, such as the propagation delays and the clock offsets. The time delay is expressed by formula (1) [19]:

$$
\tau=\tau_{g}+\tau_{c l k}+\tau_{\text {inst }}+\tau_{\text {trop }}+\tau_{\text {iono }}+\cdots,
$$

where $\tau_{g}$ is the geometric delay; $\tau_{c l k}$ is the offset of the reference clocks and the clock of each observatory; $\tau_{\text {inst }}$ is the delay of cable and equipment; $\tau_{\text {trop }}$ is the tropospheric atmosphere propagation delays; $\tau_{\text {iono }}$ is the ionospheric atmosphere propagation delays.

Each observatory transmits the observation data to a VLBI data processing center, performs data playback and cross-correlation calculation to obtain interference fringes, and then uses the cross-correlation spectrum data to calculate the time delay and delay rate of the signal arriving at each observatory. The time delay and delay rate measured by VLBI reflect the geometric relationship and variation between the radio source and the baseline. The purpose of VLBI measurement is to determine the direction vector of the radio source and the baseline vector between stations. However, the time delay of VLBI measurement is affected not only by the geometric relationship between the radio source and the baseline, but also by the atmospheric and inter-station clock differences.

In the case of absolutely calibrated cable and instrument path lengths, inter-station clock differences will be obtained when delays obtained by correlation process subtract theoretical delays such as geometric delay and atmospheric propagation delay [20]. Therefore, if the Earth's orientation, the station positions, and the ionospheric and tropospheric delays are known, VLBI can directly determine the clock difference between the two stations. With regard to station positions, the coordinates of geodetic VLBI stations can be modeled with millimeter accuracy, which can be used as prior information in time and frequency transfer. When the station position is known, the geometric delays $\tau_{g}$ can be accurately calculated. By observing two or more different frequencies, the ionosphere delays $\tau_{\text {iono }}$ can be eliminated. The tropospheric delays are related to the observation direction and atmospheric state. The tropospheric delay in any direction can be expressed by the product of the zenith tropospheric delay and mapping function. Though the mapping function is related to elevation angle, the clock difference is independent of the elevation angle; thus, the clock difference and the tropospheric delay can be separated in the estimation process [21]. When the VLBI radio telescope is deployed in the laboratory, keeping the national standard time, the deviation between the clock of other VLBI stations and the national standard time can be calculated via geodesic VLBI technology. Therefore, it is feasible to use geodesic VLBI technology for time and frequency transfer with high precision.

In some VLBI geodetic data processes and analyses, the clock offset and its change rate of each station are estimated according to the selected reference station. When analyzing the geodetic VLBI experiments conducted regularly by the IVS, the mean square error (MSE) of clock offset is usually approximately $20 \mathrm{ps}$ [22]. This precision is nearly an order of magnitude higher than GPS or TWSTFT.

\subsection{GNSS CV for time transfer}

Ever since Allan D.W. and Weiss M.A. first presented the GPS CV time transfer method at the IEEE International Frequency Control Symposium in 1980 [23], and with the development of the global satellite navigation system, GPS CV has gradually developed into GNSS CV (including GPS, GLONASS, Galileo, BeiDou, QZSS), wherein the associated technology and its applications have developed rapidly. Currently, GNSS CV is one of the main contributors to the connection, by the Bureau International des Poids et Mesures (BIPM), of more than 500 atomic clocks in more than 70 time laboratories in the world in the calculation of TAI. In 2015, a new GNSS time transfer standard was defined for the "Common Generic GNSS Time Transfer Standard (CGGTTS) Version 2E", so as to include the results corresponding to all GNSS satellites [24]. Anyone observing in standard mode can obtain highprecision CV time transfer.

The GNSS CV method connects the local clocks of the time laboratories to a GNSS time and frequency receiver. This method uses GNSS satellite clock time as a common reference source. Two remote time laboratories observe the same satellite synchronously. The difference between laboratory time and satellite clock time is measured. The relative deviation of time between the two laboratories is determined by comparing the observation results of the two laboratories [25]. The principle of GNSS CV time transfer is shown in Figure 2. Compared with the GNSS one-way timing method, the GNSS CV method can effectively eliminate the influence of satellite clock differences, weaken the influence of satellite orbit error and atmospheric delay (which is related to the distance between stations), and thus significantly improve the accuracy of remote time transfer. It has the characteristics of high precision, wide coverage, low cost, and continuous operation. The GNSS CV method is a kind of remote time transfer technology. Because of its high precision and easy operation, GNSS CV has been recently gradually applied to such fields as communication, power, transportation, highspeed digital network synchronization, national defence, and space technology to achieve long-distance clock synchronization and frequency calibration.

GNSS CV technology has developed such that from the perspective of a GNSS satellite, two atomic clocks at any two places on earth can use the time signal from the same 


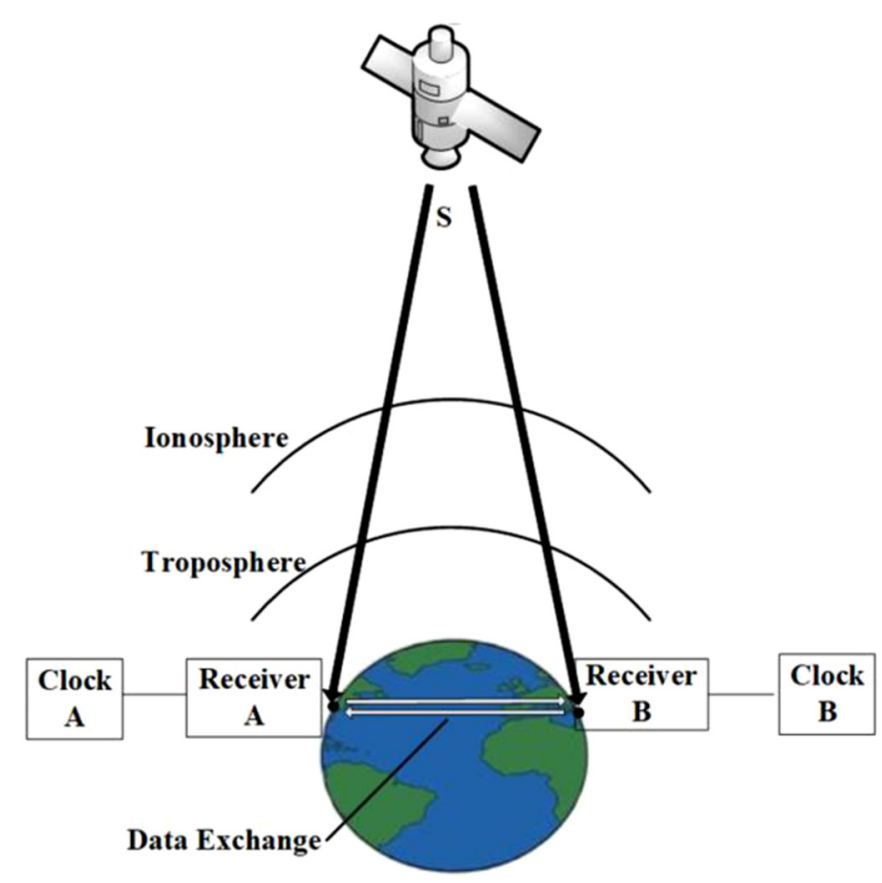

Fig. 2. Principle of GNSS common view (CV) time transfer.

satellite simultaneously to conduct time frequency comparison. Two GPS time receivers from hypothetical stations $\mathrm{A}$ and $\mathrm{B}$ under the action of the same CV time schedule receive the same satellite signal at the same time. The output second pulses by the local atomic clock are sent to the receiver's built-in counter chip; by a comparison with output second pulses by the GPS receivers, the time differences $\Delta t_{s A}$ and $\Delta t_{s B}$ between the local and satellite clock times are obtained, respectively. By subtracting the two types, the time difference between the two atomic clocks is available:

$$
\begin{aligned}
& \Delta t_{s A}=\left(t_{s}-t_{A}\right), \\
& \Delta t_{s B}=\left(t_{s}-t_{B}\right),
\end{aligned}
$$

where $\Delta t_{s A}$ is time difference between the local clock $\mathrm{A}$ and the satellite clock $\mathrm{S} ; \Delta t_{s B}$ is the time difference between the local clock $\mathrm{B}$ and the satellite clock $\mathrm{S} ; t_{s}$ is the time of the satellite $\mathrm{S}, t_{A}$ and $t_{B}$ are the times of station A's clock and station B's clock, respectively. From the difference of equations (2) and (3), the time difference is obtained [23]:

$$
\Delta t_{s A}-\Delta t_{s B}=\left(t_{s}-t_{A}\right)-\left(t_{s}-t_{B}\right)=t_{B}-t_{A}=t_{B A} .
$$

The characteristics of the GNSS CV time transfer technology eliminates the influence of satellite clock errors and weakens the influence of satellite orbit error and of the ionosphere and the troposphere. The influence of the ionosphere and the troposphere is related to the distance between stations, eliminating such negative influences more completely over shorter baselines. Furthermore, GNSS CV time transfer technology is relatively simple, inexpensive, highly-precise, and convenient to use. These attributes, together with its ability to attain a transmission precision ration of $2 \mathrm{~ns}$ [26], makes this technology the optimal method for long-distance clock comparison.

In 2011, the National Time Service Center, Chinese Academy of Sciences (NTSC) developed a precise time service system based on GNSS CV (GCVPTS) [27]. The developed system uses Internet technology to transmit the GNSS data in real time and then processes data online. It can quickly measure the relative clock difference between the user clock and the Chinese national standard time (UTC (NTSC)) and achieve the nanosecond level high precision time synchronization between the user clock and UTC (NTSC). Additionally, the GNSS CV time comparison between any two GNSS CV stations can also be achieved.

\subsection{Comparison experiments between VLBI and GPS CV}

To compare VLBI time transfer results with GPS CV time transfer, we conducted geodetic VLBI experiments using the Kunming-Urumqi baseline from 2015-2017. The Kunming station, located on Phoenix Mountain in the eastern suburbs of Kunming China, has a 40-m radio telescope. The Urumqi station, located in Nanshan of Urumqi China, has a $25-\mathrm{m}$ radio telescope. The KunmingUrumqi baseline length is approximately $2500 \mathrm{~km}$. The two stations have GNSS CV time and frequency transfer receivers which run continuously and share the hydrogen atomic clock with the VLBI observation system [28]. The experimental scheme is shown in Figure 3.

On October 22, 2015 (MJD57317), we conducted the first test experiments using the original hydrogen clock systems of Kunming station and Urumqi station, without any changes. From 2016-2017, we conducted 6 test experiments with replaced hydrogen clocks at Kunming and Urumqi stations. Here, we discuss only the MJD57317 (October 22, 2015), MJD57420 (February 02, 2016), MJD57519 (May 11, 2016), and MJD57837 (March 25, 2017 ) test experiments. The details of the data processes and analysis of GPS CV and VLBI are listed in Table 1.

\section{Results}

The ability of VLBI and GPS CV technologies using CVN VLBI Data and Joint Atomic Time of China (JATC) GPS CV data to execute time transfer was compared. To do so, two VLBI observation stations (Kunming and Urumqi), which belong to the CVN, and two GPS CV observation stations (Kunming and Urumqi), which belong to JATC network, were selected. At each station, VLBI and GPS share the same hydrogen atomic clock (as shown in Fig. 3). Therefore, in theory, the difference in the CVN between Kunming station and Urumqi station is the difference of the JATC between Kunming and Urumqi. However, time and frequency signal transmission links are different, and there are systematic differences.

Figures 4-7 show the experimental results of MJD57317, MJD57420, MJD57519, and MJD57837 time comparisons between Kunming and Urumqi using VLBI. 


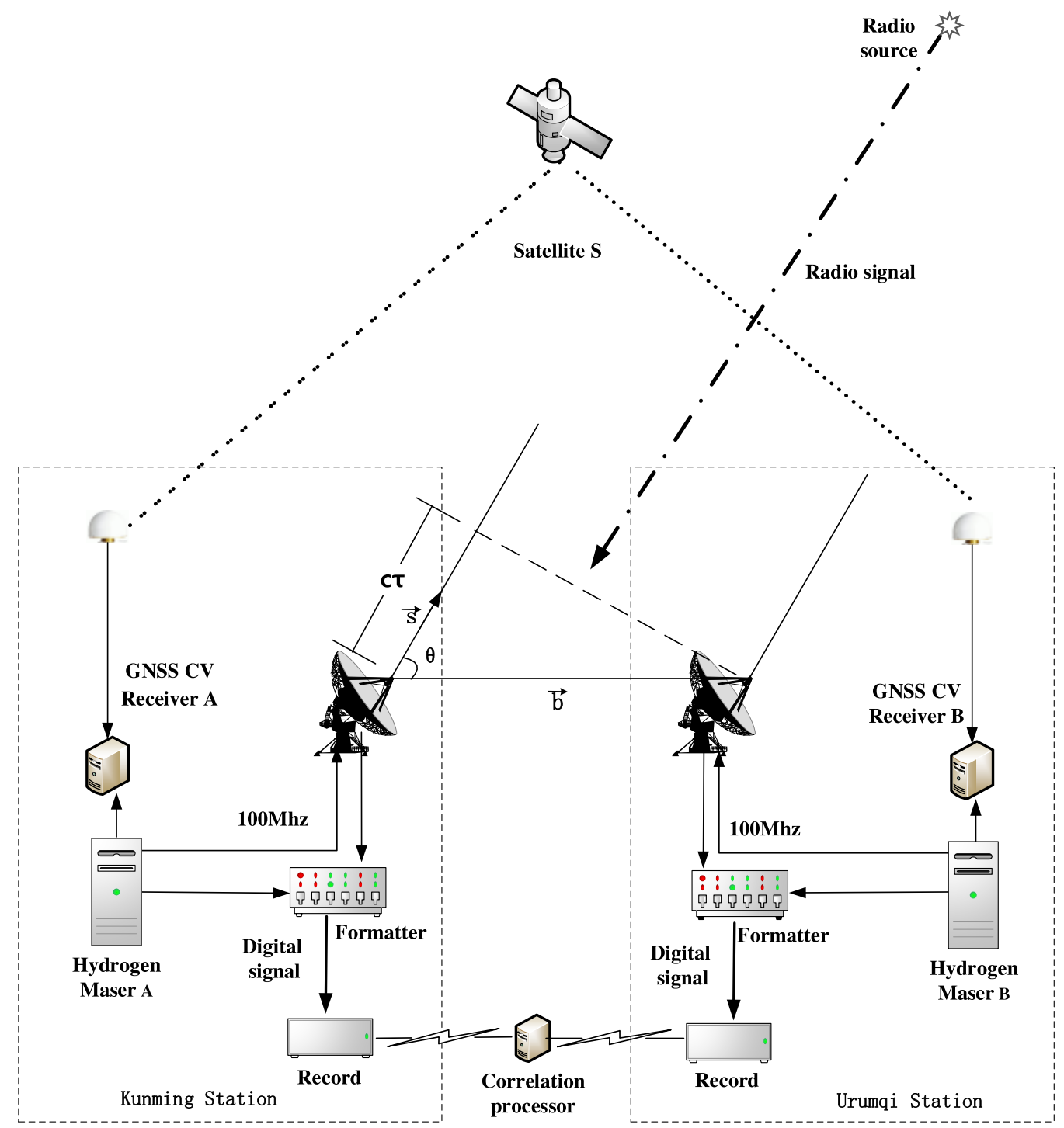

Fig. 3. Schematic diagram of a VLBI Time transfer experiment.

Table 1. Data processes and analysis of GPS CV and VLBI.

\begin{tabular}{|c|c|c|c|}
\hline Technology & Software & Strategy & Estimate \\
\hline VLBI & Calc/Solve & $\begin{array}{l}\text { Single baseline Kunming-Urumqi } \\
\text { Reference station (Shanghai) } \\
\text { X/S ionosphere-free linear combination }\end{array}$ & $\begin{array}{l}\text { Station coordinates } \\
\text { Atmospheric delay }(1 \mathrm{~h}) \\
\text { Clock offset }(1 \mathrm{~h}) \\
\text { Piecewise linear fitting }\end{array}$ \\
\hline GNSS CV & GCVPTS & GPS CV (pseudo range) & $\begin{array}{l}\text { Atmospheric delay } \\
\text { Clock (Kunming)-Clock (Urumqi) (16 min) } \\
\text { Vondrak filter }\end{array}$ \\
\hline
\end{tabular}

It can be seen that there is an obvious relative clock difference between the atomic clocks of the two stations; the clock difference has a significant linear change, with a daily variation of less than $230 \mathrm{~ns}$. Thus, the hydrogen clock equipped with VLBI station has adequate stability; however, its frequency accuracy is not high, and there is an obvious frequency deviation between the two stations. The results of inter-station clock differences obtained by the satellite $\mathrm{CV}$ method are also given. Additionally, the clock difference between the local hydrogen clock at both Kunming and Urumqi stations and the GPS time (GPST) across 20 days was recorded. 

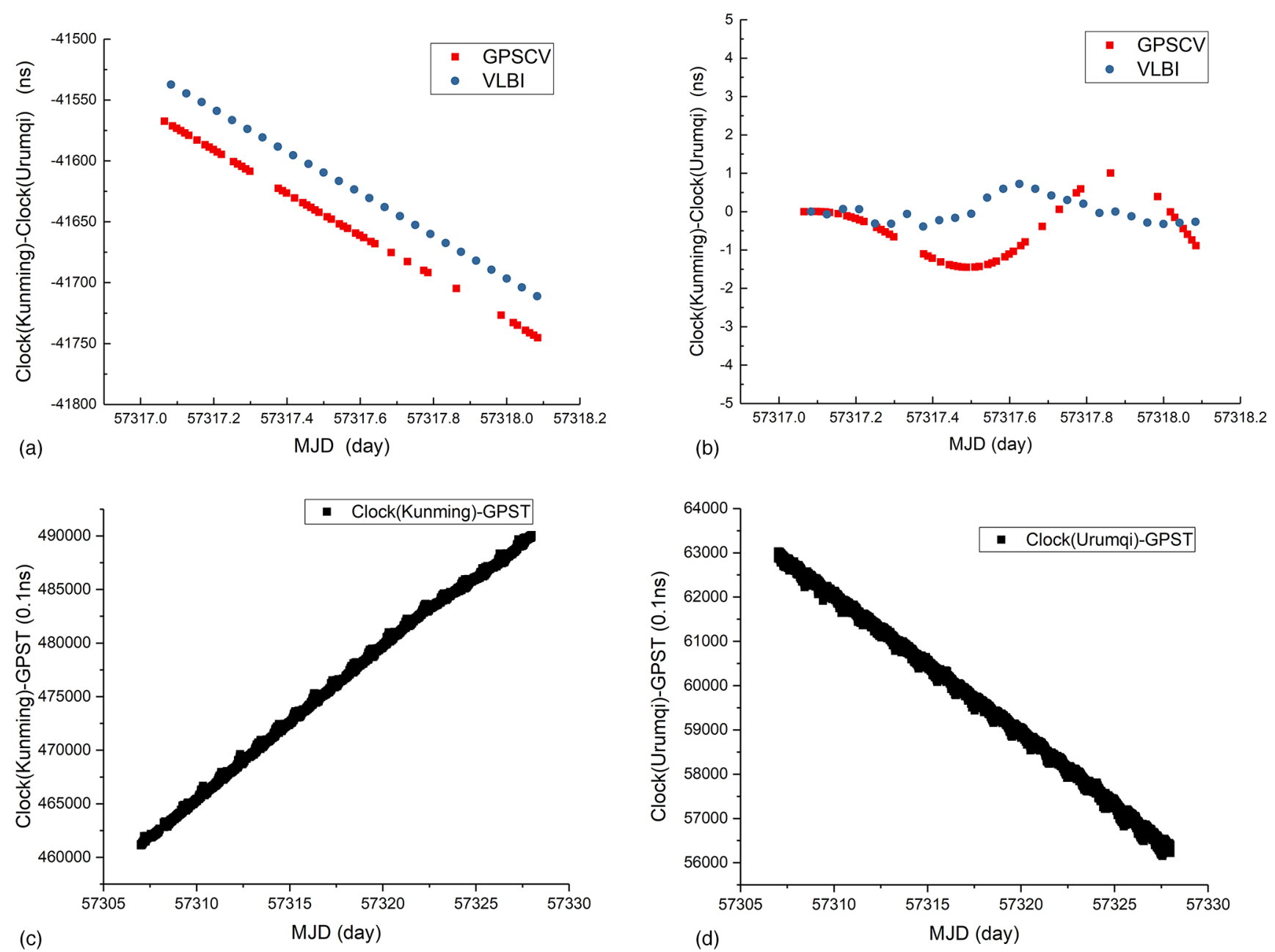

Fig. 4. Clock differences at the Kunming and Urumqi stations in MJD57317. (a) Clock differences calculations based on GPS CV and VLBI respectively. (b) Clock difference series after removing the linear trend. (c) Clock difference series between the Kunming station clock and GPST across 20 days surrounding MJD57317. (d) Clock difference series between the Urumqi station clock and GPST across 20 days surrounding MJD57317.

Figure 4 shows the clock difference series at both Kunming and Urumqi stations in MJD57317. Figure 4a presents the clock difference series calculated based on VLBI and GPS CV in MJD57317; it can be seen that the time comparison results obtained by the two technologies of GPS CV and VLBI have the same trend, however, with some obvious system differences. Though both stations involve VLBI and GPS CV technologies which share the same hydrogen atomic clock at every site, time and frequency signal transmission links are different. The time comparison results of GPS CV are corrected by timefrequency link delay, which is the actual inter-station clock difference. However, the VLBI comparison results have no system delay correction, and include the effects of transmission delay and equipment delay, which are fixed, to a certain extent, and in a short time. To further compare and analyze the details of the differences between GPS CV and VLBI results, Figure 4b shows the results after removing the linear variation; additionally, the system difference is deducted from the VLBI results. From
Figure $4 \mathrm{~b}$, it can be seen that the time comparison results obtained by GPS CV and VLBI have little differences in variation details. Figure $4 \mathrm{c}$ shows a series of clock differences between the Kunming station clock and GPST across 20 days around MJD57317; it can be seen that the hydrogen atomic clock at Kunming station was normal for these 20 days in that the hydrogen atomic clock was working steadily. Figure 4d shows a clock difference series between the clock at Urumqi station and GPST across 20 days around MJD57317; similar to the difference series at Kunming station, it can be seen that the hydrogen atomic clock at Urumqi station was normal for these 20 days.

Figure 5 shows the clock difference series of Kunming and Urumqi stations in MJD57420. Figure 5a presents the clock difference series calculations based on VLBI and GPS CV. For the convenience of curve display, the results of VLBI are moved up by $18500 \mathrm{~ns}$. It can be seen that the time comparison results obtained by the two technologies, GPS CV and VLBI, have the same trend; however, there 

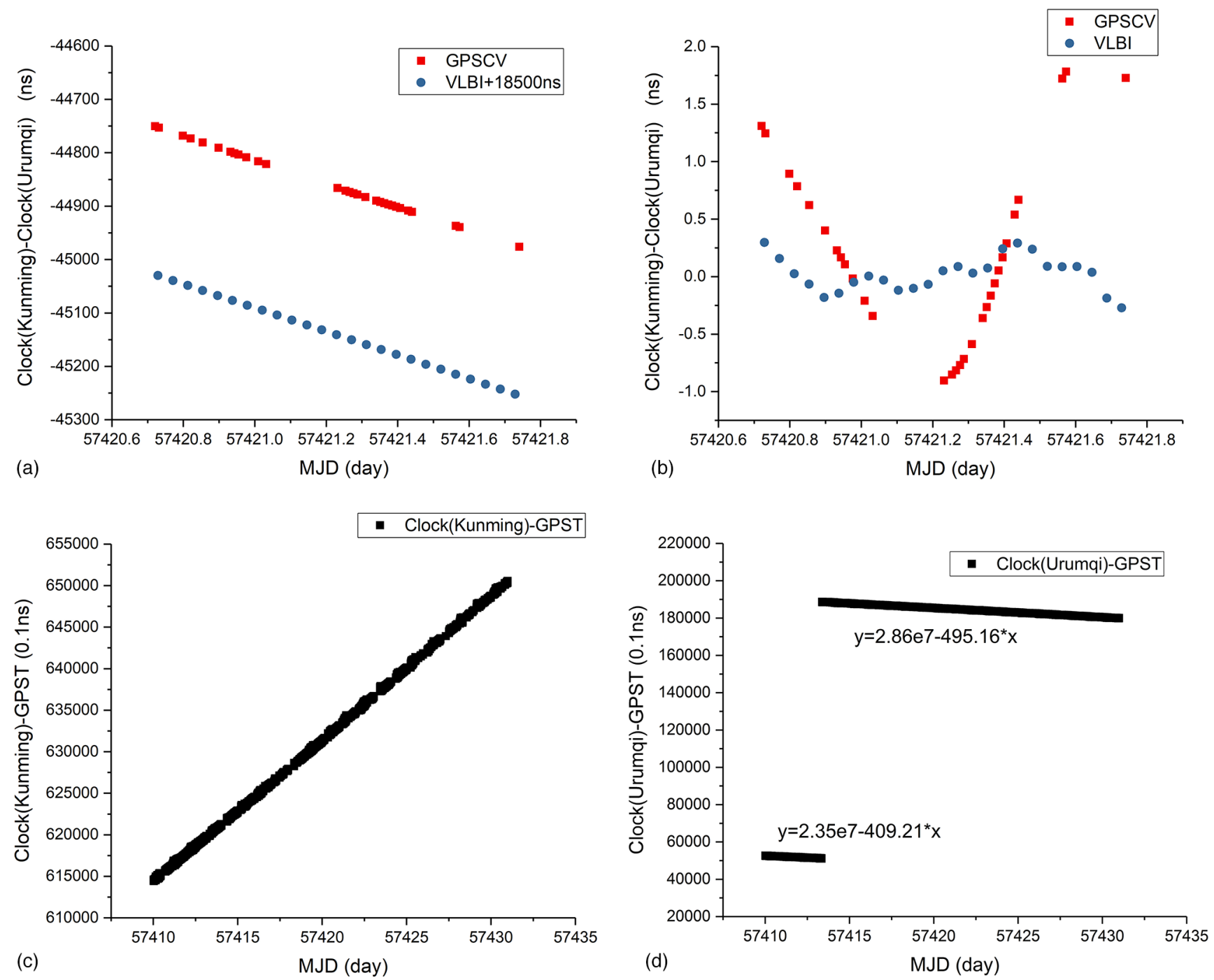

Fig. 5. Clock differences at the Kunming and Urumqi stations in MJD57420. (a) Clock differences calculations based on GPS CV and VLBI respectively. (b) Clock difference series after removing the linear trend. (c) Clock difference series between the Kunming station clock and GPST across 20 days surrounding MJD57420. (d) Clock difference series between the Urumqi station clock and GPST across 20 days surrounding MJD57420.

are obvious system differences around approximately $19000 \mathrm{~ns}(19 \mu \mathrm{s})$, which are more than the system difference of MJD57317. This can be attributed to the fact that the hydrogen clock has been replaced at Urumqi Station as shown in Figure 5d. From Figure 5c, it can be seen that the hydrogen atomic clock of the Kunming station has been normal across the 20 recorded days.

Figure 6 shows the clock difference series of Kunming and Urumqi stations for MJD57519. Figure 6a presents the clock difference series calculated based on both VLBI and GPS CV. For the convenience of curve display, the results of VLBI are moved up by $9000 \mathrm{~ns}(9 \mu \mathrm{s})$. From Figure $6 \mathrm{a}$, it can be seen that the time comparison results obtained by the two technologies have the same trend, but with obvious system differences around approximately $9 \mu \mathrm{s}$, which is more than the system difference of MJD57317 and less than the system difference of MJD57420. This can be attributed to the replaced hydrogen clock at Urumqi Station being in adjustment and not yet adjusted to an optimal state. From Figure $6 \mathrm{c}$ and $\mathrm{d}$, it can be seen that the hydrogen atomic clock of Kunming station and Urumqi station were normal for the 20 recorded days.

Figure 7 shows the clock difference series of the Kunming and Urumqi stations for MJD57837. The analysis method of MJD57837 is similar to that of the previous experiments. From Figure $7 \mathrm{c}$, it can be seen that the hydrogen atomic clock of the Kunming station was essentially normal for the 20 recorded days. From Figure $7 \mathrm{~d}$, it can be seen that the hydrogen atomic clock of the Urumqi station is discontinuous and has an obvious clock jump, though stable around MJD57837 for 4 days. 

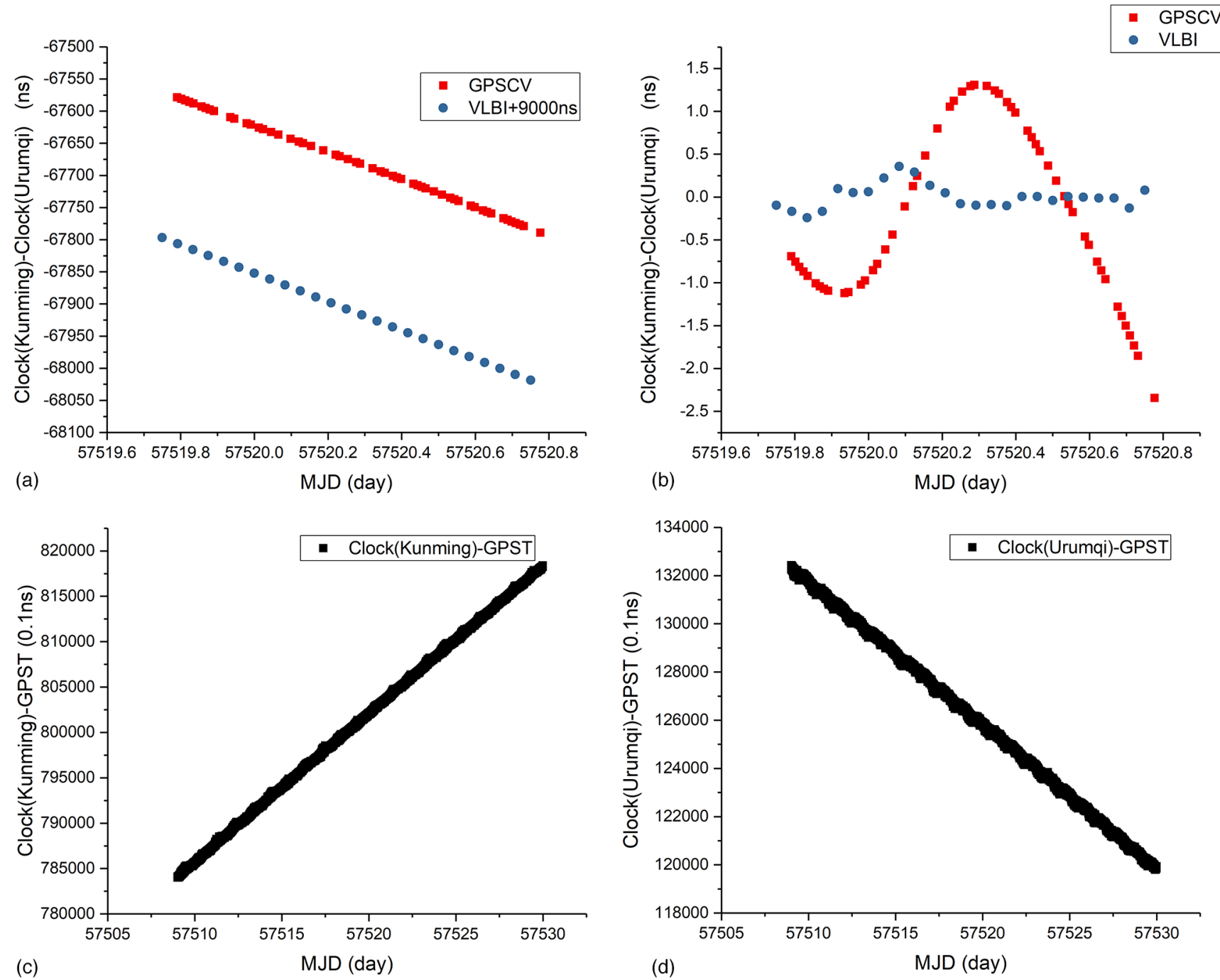

Fig. 6. Clock differences at the Kunming and Urumqi stations in MJD57519. (a) Clock differences calculations based on GPS CV and VLBI respectively. (b) Clock difference series after removing the linear trend. (c) Clock difference series between the Kunming station clock and GPST across 20 days surrounding MJD57519. (d) Clock difference series between the Urumqi station clock and GPST across 20 days surrounding MJD57519.

\section{Discussion}

Overall, the experimental results of MJD57317, MJD57420, MJD57519, and MJD57837 are largely the same. It can be seen that the time comparison results obtained by the two technologies, GPS CV and VLBI, have the same trend, though with obvious system differences. The specific statistical results are shown in Table 2.

Table 2 shows the statistical results of the time comparisons for the MJD57317, MJD57420, MJD57519, and MJD57837 experiments. From column three of Table 2 (clock difference variation), it follows that the trend of clock difference calculated by VLBI and GPS CV is essentially the same. The clock variation differences between VLBI and GPS CV technology is minimal, i.e. less than $1.0 \mathrm{~ns} /$ day, indicating that the hydrogen clocks at the two stations are stable and that the results calculated by the two techniques are extremely similar. Column four of Table 2 shows that the systematic differences of VLBI and GPS CV time comparisons. The system delay of GPS CV has been corrected for in the comparison results. Therefore, the system deviation is mainly caused by the absence of system delay correction in the VLBI comparison results, including cable transmission delay and instrument delay. These effects are fixed to a certain extent and recorded only from a short interval. However, in longer periods and with the aging and deformation of transmission cables and equipment, the transmission delay of cables will change, as will the system deviation. The abrupt increase of the system deviation for MJD57420 and MJD57519 can be attributed to the problem experienced by the hydrogen clock at Urumqi Station. Column five of Table 2 shows the standard deviation (STD) of the differences between atomic clocks 


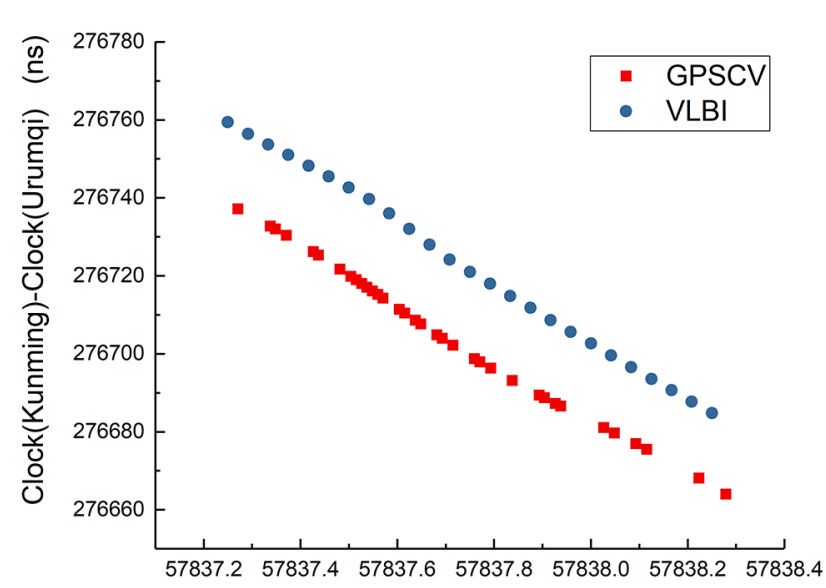

(a)

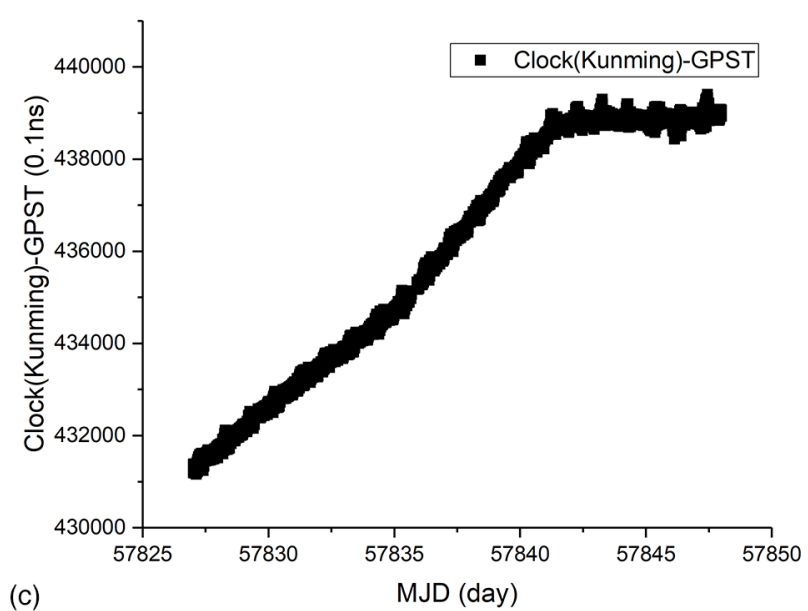

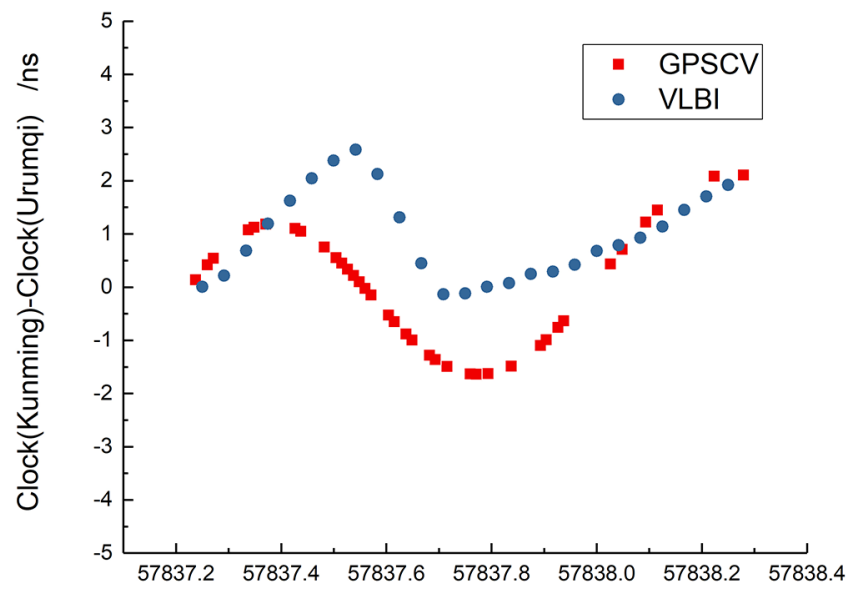

(b) MJD (day)

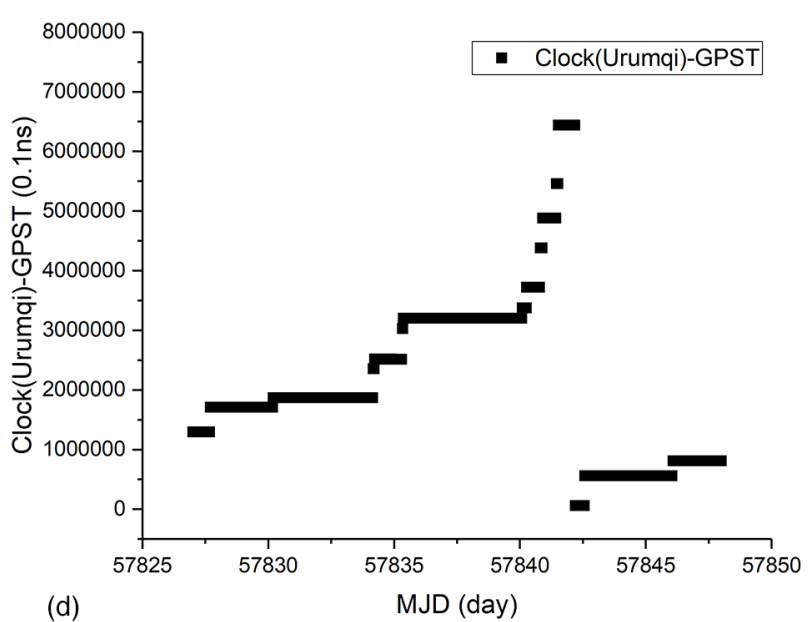

Fig. 7. Clock differences at the Kunming and Urumqi stations in MJD57837. (a) Clock differences between calculations based on GPS CV and VLBI. (b) Clock difference series after removing the linear trend. (c) Clock difference series between the Kunming station clock and GPST across 20 days surrounding MJD57837. (d) Clock difference series between the Urumqi station clock and GPST across 20 days surrounding MJD57837.

Table 2. Statistical results of time comparisons.

\begin{tabular}{lllll}
\hline MJD & Technology & Clock difference variation (ns/day) & Systematic difference (ns) & STD (ns) \\
\hline \multirow{2}{*}{57317} & VLBI & -173.5 & 722 & 0.9 \\
& GPS CV & -173.6 & 18785 & 1.7 \\
57420 & VLBI & -221.6 & & \multirow{2}{*}{1.6} \\
& GPS CV & -222.0 & 9225 & \\
57519 & VLBI & -222.1 & & \\
& GPS CV & -221.8 & 788 & \\
\hline
\end{tabular}

calculated by VLBI and GPS CV; it shows the consistency of VLBI and GPS CV in time comparisons. The precision of GPS CV is generally greater than $2 \mathrm{~ns}$. It can be seen that the clock differences between Kunming and Urumqi stations obtained by VLBI technology is essentially the same as that obtained by GPS CV technology. The difference occurs within the accuracy range of the satellite CV method. Overall, VLBI can accurately measure the inter-station clock difference of atomic clocks at two stations. 
According to the experimental results, the variation in the hydrogen atomic clock difference between Kunming and Urumqi VLBI stations obtained by VLBI technology is the same as that obtained by the satellite CV method. Differences exist within the range of accuracy of the CV method, wherein VLBI measures clock difference with higher accuracy than the CV method. However, there are obvious deviations between the results obtained by the two comparison techniques because the equipment delays in the VLBI system had not been calibrated. Though this has no effect on the results of VLBI geodesy, it does have a great influence on the calculation of inter-station clock differences.

Therefore, to achieve remote time and frequency transfer using VLBI, time-frequency link delay must be calibrated in the VLBI observation system. Moreover, the VLBI analytical model should be further studied. In the current VLBI geodesic application model, the clock difference resolution strategy constitutes a method of global constraints combined with real-time estimation. Specifically, in $24 \mathrm{~h}$, the clock difference is considered to be a quadratic polynomial function with additional piecewise linear variation.

\section{Conclusions}

To compare the results of VLBI and GNSS CV time transfer, the data of VLBI and GNSS CV have been analyzed in depth. The results show that the consistency of time comparison results based on VLBI technology and those based on the GNSS CV method are compared via observation data from Kunming Station and Urumqi Station. Analysis of this data shows that it is theoretically feasible to use VLBI for remote time and frequency transfer. However, Geodetic VLBI is usually not interested in the clock difference parameter which is as a by-product of geodetic VLBI analysis. Clock difference parameters may absorb other error terms, so the clock difference obtained is not the actual inter station clock difference. Therefore, in practical application, it is crucial to calibrate the system delay in order to achieve high accuracy time comparison. A VLBI time and frequency transfer system needs to confirm in the following three items on the system delay side. First, the formatter clock which defines the time representation for the VLBI processing by providing an arbitrary synchronized timescale to the data recording has to be calibrated and constantly measured using a reference delay measurement system to another clock or timescale. Second, delays induced by environmental changes have to be estimated. Third, the signal chain from the fiducial point of the radio antenna system to the formatter clock needs to be calibrated by measuring equipment delays or by using differential calibration techniques. Currently, accurate calibration poses the greatest challenge. Additionally, in further developing VLBI time transfer, essential areas for continuing study include both methods to avoid the influence of tropospheric model errors and solutions to clock difference constraints.
In the future, more work should continually focus on time and frequency transfer using VLBI technology. For the practical application of VLBI technology in time and frequency transfer, it can be anticipated that future VLBI system will improve VLBI's time transfer capabilities by providing higher sensitivities, all-weather operation and possible real-time capabilities. The VLBI system with broadband $24 / 7$ observations will greatly improve the link noise level, which may make high-precision remote time transfer possible. In addition, the system delay calibration, continuous correlation processing and data analysis need to be studied in depth.

This work has been supported by the National Natural Science Foundation of China (No. 11373028) and Young Innovation Talents Project of National Time Service Center, Chinese Academy of Sciences (No. Y917SC1S07). We would like to thank the Yunnan Observatory, CAS and Xinjiang Observatory, CAS for providing VLBI data. We thank the Shanghai Observatory, CAS for providing correlation processing.

\section{References}

1. BIPM, Annual Report on Time Activities, 12th edn., 10-14, 2017

2. N. Hinkley, J.A. Sherman, N.B. Phillips et al., An atomic clock with $10^{-18}$ instability, Science 341, 1215-1218 (2013)

3. T.L. Nicholson, S.L. Campbell, R.B. Hutson et al., Systematic evaluation of an atomic clock at $2 \times 10^{-18}$ total uncertainty, Nat. Commun. 6, 6896 (2015)

4. C.W. Chou, D.B. Hume, J.C. Koelemeij et al., Frequency comparison of two high-accuracy $\mathrm{Al}+$ optical clocks, Phys. Rev. Lett. 104, 070802 (2010)

5. N. Huntemann, C. Sanner, B. Lipphardt et al., Single-ion atomic clock with $3 \times 10^{-18}$ systematic uncertainty, Phys. Rev. Lett. 116, 063001 (2016)

6 . Y. Koyama, The use of very long baseline interferometry for time and frequency metrology, MAPAN - J. Metrol. Soc. India 27, 23-30 (2012)

7. J. Ray, K. Senior, Geodetic techniques for time and frequency comparisons using GPS phase and code measurements, Metrologia 42, 215-232 (2005)

8. F. Nakagawa, J. Amagai, R. Tabuchi, Y. Takahashi, M. Nakamura, S. Tsuchiya, S. Hama, Carrier-phase TWSTFT experiments using the ETS-VIII satellite, Metrologia 50, 200-207 (2013)

9. O. Lopez, A. Haboucha, B. Chanteau, C. Chardonnet, A. Amy-Klein, G. Santarelli, Ultra-stable long distance optical frequency distribution using the internet fiber network, Opt. Express 20, 23518 (2012)

10. S. Droste, F. Ozimek, T. Udem, K. Predehl, T.W. Hänsch, H. Schnatz, G. Grosche, R. Holzwarth, Optical frequency transfer over a single-span1840 km fiber link. Phys. Rev. Lett. 111, 110801 (2013)

11. M.P. Hess, J. Kehrer, M. Kufner, S. Durand, G. Heijc, H. Frühauf, L. Cacciapuoti, R. Much, R. Nasca, The ACES MWL status and test results, Proceedings of the Joint IEEE International Frequency Control Symposium and European Frequency and Time Forum, San Francisco, CA, USA, 2-5 May 2011, 26-33 
12. E. Samain et al., T2L2: time transfer by laser link, in Proceedings of the 15th Workshop on Laser Ranging, Canberra, Australia, 15-20 October 2006 (2006)

13. H. Schuh, D. Behrend, VLBI: A fascinating technique for geodesy and astrometry, J. Geodyn. 61, 68-80 (2012)

14. C. Rieck, R. Haas, K. Jaldehag, J. Johansson, VLBI time-transfer using CONT08 data, in EFTF-2010 24th European Frequency and Time Forum, Noordwijk (2010), pp. $1-8$

15. H. Takiguchi, T. Hobiger, A. Ishii, R. Ichikawa, Y. Koyama, Comparison with GPS time transfer and VLBI time transfer, IVS NICTTDC News 28, 10-15 (2007)

16. C. Rieck, R. Haas, P. Jarlemark, K. Jaldehag, VLBI frequency transfer using CONT11, European Frequency and Time Forum (EFTF) (2012), pp. 163-165

17. F. Takahashi, T. Kondo, Y. Takahashi, Y. Koyama, Very long baseline interferometer (IOS Press, Tokyo, Japan, 2000)

18. T.A. Clark, B.E. Corey, J.L. Davis, G. Elgered, T.A. Herring, H.F. Hinteregger, C.A. Knight, J.I. Levine, G. Lundqvist, C. Ma, E.F. Nesman, R.B. Phillips, A.E.E. Rogers, B.O. Rönnäng, J.W. Ryan, B.R. Schupler, D.B. Shaffer, I.I. Shapiro, N.R. Vandenberg, J.C. Webber, A.R. Whitney, Precision geodesy using the Mark-III very long baseline interferometer system, IEEE Trans. Geosci. Remote Sens. GE-23, 438-449 (1985)

19. W. Cannon, Overview of VLBI, in International VLBI Service for Geodesy and Astrometry 1999 Annual Report.
NASA/TP-1999-209243, edited by N. Vandenberg, K. Baver (1999), pp. 13-17

20. O.J. Sovers, J.L. Fanselow, C.S. Jacobs, Astrometry and geodesy with radio interferometry: experiments, models, results, Rev. Mod. Phys. 70, 1393-1454 (1998)

21. T. Hobiger, C. Rieck et al., Combining GPS and VLBI for inter-continental frequency transfer. Metrologia 52, 251-261 (2015)

22. H. Takiguchi, Y. Koyama, R. Ichikawa et al., Comparison study of VLBI and GPS carrier phase frequency transfer using IVS and IGS data, IVS NICT-TDC News 29, 23-27 (2008)

23. D.W. Allan, M.A. Weiss, Accurate time and frequency transfer during common-view of a GPS satellite, in Proceedings of the 1980 IEEE Frequency Control Symposium, Philadelphia, PA, USA, 28-30 May, 1980 (1980), pp 334-356

24. P. Defraigne, G. Petit, CGGTTS-Version 2E: an extended standard for GNSS Time Transfer, Metrologia 52, G1 (2015)

25. Z. Jiang, W. Lewandowski, Use of GLONASS for UTC time transfer, Metrologia 49, 57-61 (2012)

26. Y.P. Gao, Z.M. Wang, Y. Qi, Application of GPS CV technology to JATC, J. Time Freq. 2, 81-86 (2004)

27. P.L. Wang, H.J. Cheng, S.J. Lin, Y.P. Gao, Design and implementation of precise time service system based on GNSS CV, J. Time Freq. 35, 137-142 (2012)

28. P.L. Wang, G.L. Wang, H.B. Cai, N. Liu, H.B. Li, Y.P. Gao, The study of time comparison method based on VLBI, Chin. J. Sci. Instrum. (2019), in press

Cite this article as: Pingli Wang, Guangli Wang, Yuping Gao, Hongbing Cai, Na Liu, Comparison of VLBI and GNSS common view for time transfer, Int. J. Metrol. Qual. Eng. 10, 15 (2019) 